\title{
Plates for Color Vision Testing
}

\author{
By Joern Kuchenbecker and Dieter Broschmann
}

New York, Thieme Publishing Group, 2014, 80 pages, ISBN: 9783131754813, list price: $\$ 69.99$ (hardcover)

Plates for Color Vision Testing (ISBN: 9783131754813) was published in 2014, by Thieme Publishing Group. The authors, Joern Kuchenbecker and Dieter Broschmann, provide a thin, hand-held, easy-to-use manual for detecting and classifying colour vision deficiencies. Detailed instructions and information on the physiology, pathology, epidemiology, and genetics of colour blindness and associated disorders are clearly and concisely explained.

The book is designed for routine medical screening and provides a simple/effective tool for detecting colour vision deficiencies in any population. There are 32 colour test plates using the principles of pseudoisochromasia (distinguishing numbers and letters on a colour background) and pseudoanisochromasia (detecting characters of differing brightness on a colour background).

There is even a cut-out $\mathrm{E}$ shape for testing children and individuals who have difficulty reading or are unfamiliar with the Latin alphabet. The authors provide practical information on administering the test and interpreting results. Plates are included for blue-yellow as well as the more common red-green deficiencies.
As a result of being able to test for blue-yellow dyschromatopsia, this colour vision testing book is superior to both Ishihara (first published in 1917) and Hardy Hand Rittler (HRR) colour testing books, because the latter two tests only test for the more common red-green dyschromatopsias.

This book is intended for all ophthalmologists, optometrist, internist, technicians, and other specialists who need to evaluate an individual's colour vision for medical or occupational reasons. If one enters the term "pseudoisochromasia" as a Google search, this book, with sample colour test plates, will be accessible for more details.

The one drawback, in my opinion, is the tightly bound form rather than an easier to use Ishihara accordion type binding/display and/or the less tightly bound HRR test book. Both make testing a bit easier on the examiner. Otherwise, this book is exceptional for colour vision testing and quicker than Farnsworth-Munsell testing.

Edward M. Cohn, MD Department of Ophthalmology William Beaumont Hospital Royal Oak, Michigan, USA 